\title{
Inferior vena cava and coronary sinus obstruction after percutaneous atrial septal defect device closure requiring surgical revision
}

\author{
Alan Weng Siong Soo, MB, MRCSI, David G. Healy, MB, MRCSI, Kevin Walsh, FRCP, and Freddie Wood, FRCSI,
} Dublin, Ireland

$\mathrm{P}$ ercutaneous device closure for atrial septal defects has been gaining in popularity as a treatment modality of atrial septal defects because of the good medium-term results and lower complication rate compared with open surgical closure. There are few reported complications arising from atrial septal defect device closure. Reported complications have been limited to device embolization, thrombus formation, cardiac arrhythmia, and delivery system failures.

\section{Case Summary}

We present a 59-year-old woman with a history of decreasing exercise tolerance and deteriorating exertional dyspnea. A systolic murmur was noted on examination, which on further study with echocardiography was identified as a large $(2.4 \mathrm{~cm})$ septum secundum atrial septal defect. Right- and left-sided heart catheterization revealed a left-to-right shunt of 3.7:1 and normal coronary arteries. The atrial septal defect was closed with a $40-\mathrm{mm}$ transcatheter atrial septal defect occlusion device: the Amplatzer Septal Occluder system (AGA Medical Corporation, Golden Valley, Minn). The patient was discharged the next day.

Three weeks after the primary procedure, the patient was readmitted to the hospital with reports of exertional dyspnea, leftsided chest pain radiating down the left arm, and presyncope episodes. On examination, she was found to be hypotensive. Electrocardiogram showed new-onset, widespread T-wave inversion. Transthoracic echocardiography showed the device to have completely occluded the atrial septal defect. However, some turbulent flow was shown in the inferior vena cava, which indicated inferior vena cava stenosis. Catheterization confirmed intermittent inferior vena cava stenosis. Ultrasound and computed tomography of the abdomen were performed. Both investigations revealed that the inferior vena cava was of normal caliber and that there was no evidence of obstruction in the intra-abdominal or intrathoracic inferior vena cava. An attempt at dilating the inferior vena cava

From the Prof Eoin O'Malley National Centre for Cardiothoracic Surgery, Mater Misericordiae University Hospital, Dublin, Ireland.

Received for publication Oct 11, 2005; accepted for publication Nov 8, 2005.

Address for reprints: Alan Weng Siong Soo, MB, MRCSI, Mater Misericordiae University Hospital, Cardiothoracic Surgery, Eccles St, Dublin D2, Ireland (E-mail: alan.soo@ucd.ie).

J Thorac Cardiovasc Surg 2006;131:1405-6

$0022-5223 / \$ 32.00$

Copyright $\odot 2006$ by The American Association for Thoracic Surgery doi:10.1016/j.jtcvs.2005.11.055

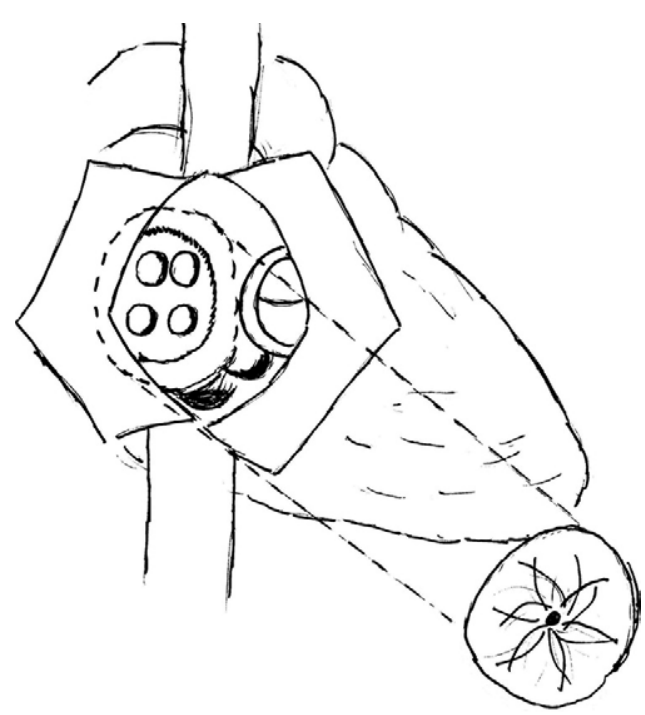

Figure 1. Impression by author showing position of low septum secundum atrial septal defect and position of device.

was performed with catheterization. This made no improvement, and the patient's condition deteriorated.

Surgical exploration was advised. At operation the inferior rim of the device was seen clearly, partially occluding the inferior vena cava and coronary sinus (Figure 1).

The device was then removed (Figure 2), leaving a large septum secundum atrial septal defect that was closed using a pericardial patch. The patient's postoperative care was uneventful, and her symptoms resolved completely. She remains symptomfree at the 3-month follow-up.

\section{Comment}

Traditionally, atrial septal defect has been repaired surgically with good long-term results. ${ }^{1}$ However, with the advent of the Amplatzer Septal Occluder device, secundum atrial septal defect is increasingly being repaired percutaneously with such a device. Repairing atrial septal defect percutaneously has proved to be safe and effective, and has good-medium term results in a pediatric population. Experience is limited in adults, although early evidence shows the procedure to be safe and effective. ${ }^{2}$ In comparison with surgical repair, percutaneous closure of atrial septal defect has a comparable success rate, lower morbidity, and reduced hospital stay in the short term. Long-term results are awaited. ${ }^{3}$ However, complications such as device malposition, residual shunts, hemolysis, embolization of device, partial occlusion of superior vena 

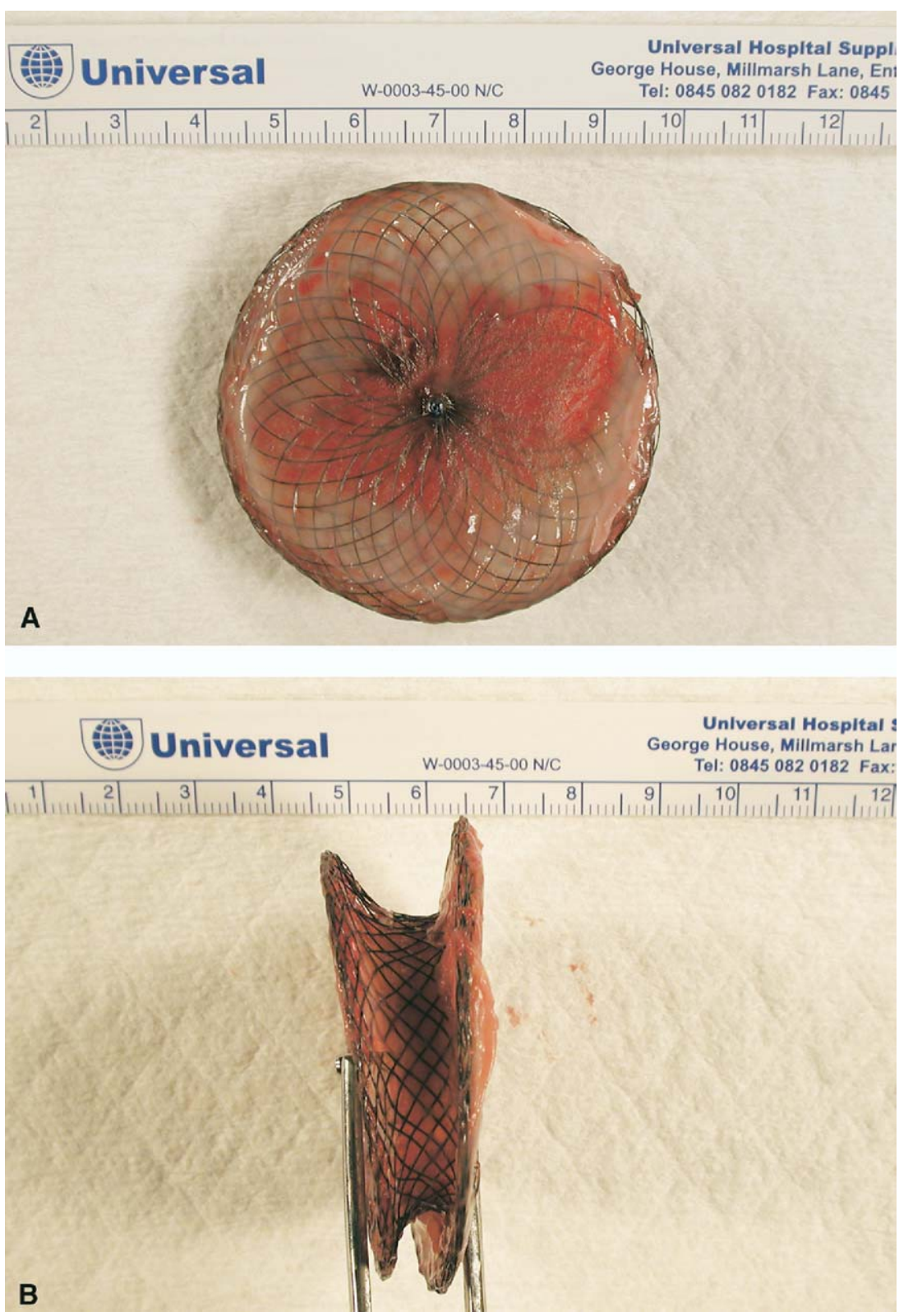

Figure 2. Explanted Amplatzer Septal 0ccluder system (AGA Medical Corporation, Golden Valley, Minn). cava, damage to mitral or tricuspid valves, cardiac perforation, and thrombus formation have all been reported. ${ }^{4}$ These complications usually require open surgical repair, although there is an increasing trend to retrieve the embolized device percutaneously. ${ }^{5}$

Devices such as the Amplatzer Septal Occluder system are still undergoing postmarket surveillance. The role of these devices is increasingly being expanded. Newer roles include closure of aorto-atrial fistulas and ruptured sinus of Valsalva aneurysm. Because the role of these devices is frequently being reevaluated, both the interventional cardiologist and cardiothoracic surgeon who work in units where these devices are being used should be aware of the potential complications and management.

\section{References}

1. Hopkins A, Bert A, Buchholz B, Guarino K, Meyers M. Surgical patch closure of atrial septal defect. Ann Thorac Surg. 2004;77:2144-50.

2. Purcell IF, Brecker SJ, Ward DE. Closure of defects of the atrial septum in adults using the Amplatzer device: 100 consecutive patients in a single center. Clin Cardiol. 2004;27:509-13.

3. Du ZD, Hijazi ZM, Kleinman CS, Silverman NH, Larntz K. Comparison between transcatheter and surgical closure of secundum atrial septal defect in children and adults: results of a multicenter nonrandomized trial. J Am Coll Cardiol. 2002;39:1836-44.

4. Preventza O, Sampath-Kumar S, Wasnick J. Late cardiac perforation following transcatheter atrial septal defect closure. Ann Thorac Surg. 2004;77:1435-7.

5. Levi DS, Moore JW. Embolization and retrieval of the Amplatzer septal occluder. Catheter Cardiovasc Interv. 2004;61:543-7. 